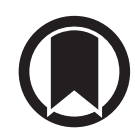

CrossMark

\title{
WHO 2019 guidelines on drug-resistant tuberculosis treatment: based on evidence or expert opinion?
}

\author{
To the Editor:
}

Since 2011, the World Health Organization (WHO) has used the Grading of Recommendations Assessment, Development, and Evaluation (GRADE) system for grading quality of evidence $[1,2]$ to update guidelines for the management of drug-resistant tuberculosis (DR-TB). This represents an undeniable improvement towards evidence-based recommendations compared to previous guidelines, which were primarily based on expert opinion [3]. However, some aspects of guideline development remain unclear. In our opinion, the WHO 2019 consolidated guidelines on DR-TB treatment [4] have raised some concerns in the way recommendations were drawn from the evidence evaluated to answer specific PICO (Patient, Intervention, Comparator, Outcome) questions.

Firstly, the evidence that was graded to assess the standardised shorter multidrug-resistant tuberculosis regimen (SCR) (PICO 1) used the 2011 [5] or 2016 [6, 7] WHO longer regimens as a comparator. This does not provide conclusive evidence on the performance of the SCR as compared to the new longer regimens that include new core drugs, such as bedaquiline and linezolid, as recommended by the 2019 WHO guideline. Similarly, the phase III trial STREAM, which tested the SCR regimen, did not include these drugs in the control arm [8]. Therefore, the recommendation of the SCR as an equal alternative to the longer regimen designed according to 2019 guidelines is based on no direct evidence.

Secondly, the analyses conducted on patients treated with the 2016 WHO longer regimen do not inform about the optimal number of drugs and the duration of the newly recommended regimen (PICO 3 and PICO 4, 5 and 6). Moreover, assessing treatment duration from retrospective observational studies, as conducted for the individual patient data meta-analysis [9] where, for instance, deaths and patients lost to follow-up were excluded from the analyses, may lead to formulating recommendations that are inherently biased. Any recommendation based on these findings would benefit from being presented as extrapolations and expert opinion. This also applies to the recommendation to routinely stop bedaquiline treatment after 6 months, which is not supported by evidence, in contrast with available observational reports $[10,11]$, and inconsistent with the recommendation to use other drugs (e.g. linezolid) for a longer duration than in their approved labelling.

Thirdly, the 2019 guidelines follow the assumption that the combination of the most effective drugs (three group A and one group B), as identified by the individual patient data meta-analysis (PICO 2), would result in the most effective longer regimen. Conversely, a similar assumption has not been made for the SCR, a regimen that excludes two of the most potent group A drugs (linezolid and bedaquiline). Furthermore, an exception was made to this second assumption with the replacement of kanamycin by amikacin in the SCR, although such a change is not supported by evidence.

We understand that some PICO questions could not be directly answered and that recommendations had to be based on extrapolations from indirect evidence; however, this needs to be made explicit to programmes and clinicians so they may be empowered to make appropriate, informed clinical decisions. As evidence evolves in the complex field of DR-TB, and with the availability of new observational and clinical trial data, it is key that guidelines make a clear distinction between evidence, expert opinion and assumptions, so as to allow appreciation of the uncertainty surrounding the recommendations.

@ERSpublications

World Health Organization guidelines on drug-resistant tuberculosis should make a clear distinction between evidence, expert opinion and assumptions to allow appreciation of the uncertainty around the recommendations http://bit.ly/2FHxmyW

Cite this article as: Guglielmetti L, Huerga H, Khan U, et al. WHO 2019 guidelines on drug-resistant tuberculosis treatment: based on evidence or expert opinion? Eur Respir J 2020; 55: 1901935 [https://doi. org/10.1183/13993003.01935-2019]. 
Lorenzo Guglielmetti $\odot^{1,2}$, Helena Huerga ${ }^{3}$, Uzma Khan $^{4}$ and Francis Varaine ${ }^{1}$

${ }^{1}$ Médecins Sans Frontières, Paris, France. ${ }^{2}$ APHP, Sorbonne Université, Site Pitié-Salpêtrière, Centre National de Référence des Mycobactéries et de la Résistance des Mycobactéries aux Antituberculeux, Sorbonne Université, INSERM, U1135, Centre d'Immunologie et des Maladies Infectieuses, Cimi-Paris, équipe 2, Paris, France. ${ }^{3}$ Epicentre, Paris, France. ${ }^{4}$ Interactive Research and Development, Dubai, UAE.

Correspondence: Lorenzo Guglielmetti, MSF France, Medical Department, 14-34 avenue Jean Jaurès, 75019 Paris, France. E-mail: lorenzo.guglielmetti@paris.msf.org

Received: 1 Oct 2019 | Accepted after revision: 19 Dec 2019

Author contributions: L. Guglielmetti and F. Varaine made a substantial contribution to the conception of the work, wrote the manuscript, critically revised the manuscript for important intellectual content, and gave final approval of the current version to be published. H. Huerga and U. Khan critically revised the manuscript for important intellectual content and gave final approval of the current version to be published.

Conflict of interest: L. Guglielmetti is co-principal investigator of endTB and endTB-Q clinical trials. H. Huerga has nothing to disclose. U. Khan has nothing to disclose. F. Varaine is director of the endTB project for MSF.

\section{References}

1 World Health Organization. WHO handbook for guideline development. 2nd Edn. Geneva, WHO, 2014. Available from: www.who.int/publications/guidelines/handbook_2nd_ed.pdf

2 Guyatt GH, Oxman AD, Vist GE, et al. GRADE: an emerging consensus on rating quality of evidence and strength of recommendations. BMJ 2008; 336: 924-926.

3 Caminero JA, García-Basteiro AL, Rendon A, et al. The future of drug-resistant tuberculosis treatment: learning from the past and the 2019 World Health Organization consolidated guidelines. Eur Respir J 2019; 54: 1901272.

4 World Health Organization. WHO Consolidated Guidelines on Drug-resistant Tuberculosis Treatment. WHO/ CDS/TB/2019.7. Geneva, WHO. 2019. Available from: https://apps.who.int/iris/bitstream/handle/10665/311389/ 9789241550529-eng.pdf

5 World Health Organization. Guidelines for the Programmatic Management of Drug-resistant Tuberculosis - 2011 Update. WHO/HTM/TB/2011.6. Geneva, WHO, 2011.

6 World Health Organization. WHO Treatment Guidelines for Drug-resistant Tuberculosis. WHO/HTM/TB/ 2016.04. Geneva, WHO. 2016.

7 Falzon D, Schünemann HJ, Harausz E, et al. World Health Organization treatment guidelines for drug-resistant tuberculosis, 2016 update. Eur Respir J 2017; 49: 1602308.

8 Nunn AJ, Phillips PPJ, Meredith SK, et al. A trial of a shorter regimen for rifampin-resistant tuberculosis. $N$ Engl Med 2019; 380: 1201-1213.

9 Ahmad N, Ahuja SD, Akkerman OW, et al. Treatment correlates of successful outcomes in pulmonary multidrug-resistant tuberculosis: an individual patient data meta-analysis. Lancet 2018; 392: 821-834.

10 Guglielmetti L, Jaspard M, Le Dû D, et al. Long-term outcome and safety of prolonged bedaquiline treatment for multidrug-resistant tuberculosis. Eur Respir J 2017; 49: 1601799.

11 Hewison C, Bastard M, Khachatryan N, et al. Is 6 months of bedaquiline enough? Results from the compassionate use of bedaquiline in Armenia and Georgia. Int J Tuberc Lung Dis 2018; 22: 766-772.

\section{WHO guidance on multidrug-resistant tuberculosis treatment: using and communicating the evidence}

From the authors:

L. Guglielmetti and co-workers acknowledge that methods used by the World Health Organization (WHO) to develop guidance have improved policies on multidrug-resistant tuberculosis (MDR-TB) in recent years. However, they express some concerns about the interpretation and communication of evidence in the 2019 WHO consolidated guidelines for drug-resistant TB treatment [1].

@ERSpublications

WHO MDR-TB treatment guidelines detail how evidence and other considerations are used to develop recommendations http://bit.ly/35qSrZT

Cite this article as: Falzon D, Schünemann HJ, Zignol M, et al. WHO guidance on multidrug-resistant tuberculosis treatment: using and communicating the evidence Eur Respir J 2020; 55: 1902325 [https://doi. org/10.1183/13993003.02325-2019]. 
WHO guidance on MDR-TB treatment remains in high demand, given the complexity of the intervention, divergent expert views on optimal therapeutic options, rapid recent developments and a shortage of reliable evidence. Since 2010, WHO has used the GRADE approach (Grading of Recommendations Assessment, Development, and Evaluation) to increase the clarity of its MDR-TB treatment guidance [1, 2], presenting estimates of effect alongside other considerations critical to successful scale-up, such as patient or caregiver preference, acceptability, feasibility, resource implications and impact on equity [3]. GRADE provides for downgrading the certainty of evidence when indirect comparisons and extrapolations are used; to be differentiated from expert opinion provided in the absence of a distinct evidence base [4]. The GRADE evidence-to-decision framework (table 1) allows guidelines expert panels convened by WHO to systematically document their decisions, to communicate uncertainties in the evidence arising from data shortcomings, and to propose research priorities (Annex 8 in [5]).

Given the rarity of randomised controlled trials, much of the current WHO MDR-TB treatment guidance relies on analyses of observational studies of standardised 9- to 11-month shorter MDR-TB regimens and individualised longer regimens of 18 months or more in widespread use. Ahead of the 2019 update of the consolidated guidelines, WHO made a call for data in an effort to exploit more contemporary practices and reduce delays incurred when analyses are restricted to published studies recovered by traditional systematic reviews [6]. New recommendations on optimal number of medicines and their duration of use in longer regimens drew upon multi-country experiences since the mid-1990s of combinations of older agents, such as fluoroquinolones and clofazimine used "off-label", as well as new medicines, such as bedaquiline. The dataset used for the guidelines update included many patients on medicines that would be strongly recommended in the new guidelines: nearly 2000 patients were on bedaquiline, 1600 on linezolid and over 3000 on moxifloxacin. The meta-analysis of these data allowed the effects of individual medicines to be compared. Among patients on bedaquiline, only 5\% received it for longer than the 6-month period for which its market authorisation had been granted. No recommendation to use bedaquiline beyond the manufacturer's indication could be entertained without clarity about the balance of benefits to harms of doing so. This is nonetheless an area where more research about the efficacy and toxicity of this medicine are expected to influence future guidance.

The limitations of methods used to associate patient outcome with treatment duration in retrospective observational studies have been described repeatedly since the first individual patient data meta-analysis to inform WHO MDR-TB guidelines was conducted [7, 8]. Until direct evidence from regimens of different durations becomes available it is unlikely that this inherent constraint will be solved by different analytical approaches alone.

Since its first release in 2016, the WHO recommendation for the shorter MDR-TB regimen was based upon studies from various settings of standardised treatments whose composition and length were near-identical to those recommended. The recommendation applies primarily to patient inclusion criteria adopted by the studies, for whom evidence of benefit and harms exists. It was not possible to compare the shorter regimen with longer regimens containing linezolid or bedaquiline, given that such regimens have until recently been largely reserved for patients who were ineligible for the shorter regimen (e.g. fluoroquinolone-resistant MDR-TB strains). The replacement of kanamycin by amikacin was always a permissible adaptation to the shorter regimen, premised upon the similarity of action of these aminoglycosides and some empirical evidence. When, in 2018, evidence from longer regimens showed

TABLE 1 Main criteria of GRADE (Grading of Recommendations Assessment, Development, and Evaluation) evidence-to-decision framework

Is the problem a priority?

How substantial are the desirable anticipated health effects?

How substantial are the undesirable anticipated health effects?

What is the overall certainty of the evidence of the intervention on health effects?

Is there important uncertainty about or variability in how much people value the main outcomes?

Do the desirable effects outweigh the undesirable health effects?

How large are the resource requirements?

What is the certainty of the evidence of resource requirements?

Are the net benefits worth the incremental cost?

What would be the impact on health equity?

Is the intervention acceptable to key stakeholders?

Is the intervention feasible to implement?

Modified from [3] with permission from the publisher. 
amikacin to be superior to kanamycin, it was recommended to use only amikacin in shorter regimens. More radical changes, such as replacing the injectable agent with bedaquiline or linezolid, could not be recommended given that no study had tested such radical departures at the time $[9,10]$. Results from MDR-TB patients treated with all-oral shorter regimens have only recently become available and are currently being evaluated by WHO.

In conclusion, GRADE frameworks communicate the evidence alongside other considerations used by WHO expert panels to determine the strength, wording and direction of evidence-based MDR-TB treatment recommendations. Analysis of pooled data has allowed the continued consolidation of recent evidence to inform global treatment policy in a context of minimal trial evidence. WHO welcomes more research and better data and commits to keep reviewing new evidence so that its global policies will benefit MDR-TB patients from shorter, safer and more effective treatment options.

Dennis Falzon ${ }^{1}$, Holger J. Schünemann $\oplus^{2}$, Matteo Zignol ${ }^{1}$, Fuad Mirzayev ${ }^{1}$ and Tereza Kasaeva ${ }^{1}$

${ }^{1}$ Global TB Programme, World Health Organization, Geneva, Switzerland. ${ }^{2}$ Dept of Health Research Methods, Evidence and Impact, and Dept of Medicine, McMaster University, Hamilton, ON, Canada.

Correspondence: Dennis Falzon, Global TB Programme, World Health Organization, 20 Avenue Appia, Geneva, Switzerland. E-mail: falzond@who.int

Received: 4 Dec 2019 | Accepted after revision: 11 Dec 2019

Author contributions: D. Falzon developed a first draft of the manuscript and the other authors proposed revisions towards its finalization. All authors agreed with the version submitted for publication. D. Falzon, T. Kasaeva, F. Mirzayev and M. Zignol are staff members of the World Health Organization (WHO). They alone are responsible for the views expressed in this publication and they do not necessarily represent the decisions or policies of WHO. The designations used and the presentation of the material in this publication do not imply the expression of any opinion whatsoever on the part of WHO concerning the legal status of any country, territory, city or area, or of its authorities, nor concerning the delimitation of its frontiers or boundaries.

Conflict of interest: D. Falzon has nothing to disclose. H.J. Schünemann declares that his employer (McMaster University) received funding from WHO related to work he carries out on guideline development on tuberculosis. $\mathrm{He}$ has also received consultancy fees from WHO for work unrelated to tuberculosis. M. Zignol has nothing to disclose. F. Mirzayev has nothing to disclose. T. Kasaeva has nothing to disclose.

\section{References}

1 World Health Organization. WHO Consolidated Guidelines on Drug-resistant Tuberculosis Treatment. WHO/ CDS/TB/2019.7. Geneva, WHO, 2019. Available from: https://apps.who.int/iris/bitstream/handle/10665/311389/ 9789241550529-eng.pdf

2 Falzon D, Jaramillo E, Schünemann HJ, et al. WHO guidelines for the programmatic management of drug-resistant tuberculosis: 2011 update. Eur Respir J 2011; 38: 516-528.

3 Moberg J, Rosenbaum S, Schünemann HJ, et al. The GRADE Evidence to Decision (EtD) framework for health system and public health decisions. Health Res Policy Syst 2018; 16: 45.

4 Schünemann HJ, Zhang Y, Oxman AD. Distinguishing opinion from evidence in guidelines. BMJ 2019; 366: 14606.

5 World Health Organization. WHO Consolidated Guidelines on Drug-resistant Tuberculosis Treatment. Annexes 3-9. WHO/CDS/TB/2019.3. Geneva, WHO, 2019. Available from https://apps.who.int/iris/bitstream/handle/10665/ 311390/WHO-CDS-TB-2019.3-eng.pdf

6 World Health Organization. Public Call for Individual Patient Data on Treatment of Rifampicin and Multidrug-resistant (MDR/RR-TB) Tuberculosis. www.who.int/tb/features_archive/public_call_treatment_RR_MDR_ TB/en/ Date last updated: 16 Feb 2018. Date last accessed: 27 Feb 2018.

7 Ahuja SD, Ashkin D, Avendano M, et al. Multidrug resistant pulmonary tuberculosis treatment regimens and patient outcomes: an individual patient data meta-analysis of 9,153 patients. PLoS Med 2012; 9: e1001300.

8 Ahmad N, Ahuja SD, Akkerman OW, et al. Treatment correlates of successful outcomes in pulmonary multidrug-resistant tuberculosis: an individual patient data meta-analysis. Lancet 2018; 392: 821-834.

9 Nunn AJ, Phillips PPJ, Meredith SK, et al. A trial of a shorter regimen for rifampin-resistant tuberculosis. $N$ Engl J Med 2019; 380: 1201-1213.

10 Ahmad Khan F, Salim MAH, du Cros P, et al. Effectiveness and safety of standardised shorter regimens for multidrug-resistant tuberculosis: individual patient data and aggregate data meta-analyses. Eur Respir J 2017; 50: 1700061 .

The content of this work is copyright of the authors or their employers. Design and branding are copyright (c)ERS 2020. 